\title{
Fatores relacionados à capacidade física de membros superiores e inferiores de idosos quilombolas
}

\author{
Factors related to the physical capacity of upper and lower limbs \\ in quilombola elderly people
}

Brenda Natally Soares Furtado (https://orcid.org/0000-0002-2570-8799) ${ }^{1}$

Ricardo Alves de Olinda(http://orcid.org/0000-0002-0509-8428) 2

Gabriela Maria Cavalcanti Costa (https://orcid.org/0000-0003-4466-6886) ${ }^{3}$

Tarciana Nobre de Menezes (https://orcid.org/0000-0003-1784-3218) ${ }^{4}$

${ }^{1}$ Programa de Pós-

Graduação em Saúde

Pública, Universidade

Estadual da Paraíba. Av.

Baraúnas 351, Campus

Universitário. 58109-753

Campina Grande PB Brasil.

brenda.natally@gmail.com

2 Departamento de

Estatística, Universidade

Estadual da Paraíba.

Campina Grande PB Brasil.

${ }^{3}$ Departamento de

Enfermagem, Universidade

Estadual da Paraíba.

Campina Grande PB Brasil.

${ }^{4}$ Departamento de

Fisioterapia, Universidade

Estadual da Paraíba.

Campina Grande PB Brasil.
Abstract This paper aimed to assess the factors related to the physical capacity of upper and lower lim$b s$ in quilombola older adults. This is a cross-sectional, analytic study performed with elderly residents in the quilombola community Caiana dos Crioulos, Alagoa Grande, Paraíba, Brazil. The researched dependent variables were the physical capacity of upper and lower limbs. It was performed the Spearman correlation and multiple and linear simple regression (95\% CI; $p<0.05$ ). This work was performed per Resolution 466/2012 from National Health Council. Forty-three older adults were assessed. The bivariate analyses showed a positive correlation between handgrip strength (HGS) and physical activity (PA), between HGS and arm muscle circumference $(A M C)$, and an inverse correlation between Short Physical Performance Battery (SPPB) and age. The multiple-model for HGS was significantly correlated with age, $A M C$, and years of study $\left(R^{2}=0.374\right.$; $p<0.05)$. Regarding the $S P P B$, it showed a significant correlation with age $\left(R^{2}=0.2524 ; p=0.001\right)$. Therefore, the muscle mass reserve, years of study, and age were factors related to the physical capacity among quilombola older adults. The strict compliance of these aspects and the early intervention can preserve physical capacity and ensure the productive inclusion and economic autonomy so much sought in the communities' social agenda.

Key words Elderly health, Physical functional performance, Ethnic groups, African Continental Ancestry Group
Resumo Objetivou-se avaliar os fatores relacionados à capacidade física de membros superiores $e$ inferiores em idosos quilombolas. Estudo transversal e analítico, realizado com a população idosa residente na comunidade quilombola Caiana dos Crioulos, Alagoa Grande, estado da Paraíba. As variáveis dependentes investigadas foram capacidade física de membros superiores e de membros inferiores. Foi realizada correlação de Spearman e regressão linear simples e múltipla (IC95\%; $p<0,05)$. O estudo foi realizado em conformidade com a Resolução 466/2012 do Conselho Nacional de Saúde do Ministério da Saúde. Foram avaliados 43 idosos. A análise bivariada mostrou correlação positiva entre FPM e PAF; entre FPM e CMB e correlação inversa entre a SPPB e a idade. O modelo múltiplo para FPM mostrou correlação significativa com a idade, $C M B$ e anos de estudo $\left(R^{2}=0,374 ; p<0,05\right)$. Para a $S P P B$ mostrou correlação significativa com a idade $\left(R^{2}=0,2524 ; p=0,001\right)$. Assim, a reserva de massa muscular, anos de estudo e idade foram fatores relacionados à capacidade física entre os idosos quilombolas. A observância criteriosa destes aspectos e a intervenção precoce podem preservar a capacidade física e garantir a inclusão produtiva e a autonomia econômica tão pleiteada na agenda social das comunidades.

Palavras-chave Saúde do idoso, Desempenho físico funcional, Grupos étnicos, Grupo com Ancestrais do Continente Africano 


\section{Introdução}

O termo capacidade está relacionado à aptidão de um indivíduo para realizar uma tarefa ou uma ação, em um ambiente uniforme e padrão ${ }^{1}$. A avaliação da capacidade física consiste em uma ferramenta importante para avaliar o estado de saúde de idosos, dada a sua relação com a funcionalidade deste grupo ${ }^{2}$. Alterações na funcionalidade dos membros inferiores ocasionam a limitação na mobilidade, que comumente conduz à incapacidade física e à perda da independência funcional ${ }^{3,4}$. Alterações da capacidade física de membros superiores podem ser importantes indicadores da força geral, preditores de doenças cardiometabólicas, incapacidade, morbidade e mortalidade precoce ${ }^{4}$.

A promoção de saúde do idoso e as ações em saúde devem considerar a funcionalidade global, capacitando o indivíduo a gerenciar a própria vida $^{5}$. Essa perspectiva amplia a atenção à saúde do idoso, retirando o foco da doença e do biológico e conduzindo para abordagens socioculturais, promovendo o envelhecimento ativo e saudável ${ }^{6}$.

Diante da importância desta abordagem, insere-se a população quilombola, que em seus fatores contextuais apresenta condições de vulnerabilidade para doenças que incluem os aspectos sociais. Essa situação de vulnerabilidade foi reconhecida pelo governo brasileiro com a criação da Secretaria Especial de Políticas de Promoção de Igualdade Racial (SEPPIR) e instituição da Política Nacional de Saúde Integral da População Negra (PNSIPN) ${ }^{7}$. As comunidades quilombolas são formadas por indivíduos com ancestralidade africana, que se destacam entre a população negra por serem mais suscetíveis em razão das diferenças socioeconômicas e sua localização geográfica majoritariamente rural $^{8}$. Essa população enfrenta dificuldades na operacionalização das políticas públicas e interrupção das ações propostas pelo Estado que favorece ao aumento das iniquidades desfavorecendo os grupos vulneráveis?.

São poucos os estudos na literatura científica que avaliam as capacidades físicas de quilombolas $^{10,11}$. Estudo realizado em duas comunidades quilombolas de Tocantins avaliou a força de preensão manual de idosas, assim como a composição corporal e a velocidade de marcha. Neste estudo verificou-se valores médios de capacidades físicas adequados $(21,79 \pm 5,57 \mathrm{Kg}$ de FPM e 1,28 $\pm 0,4 \mathrm{~m} / \mathrm{s}$ de velocidade de marcha $)^{10}$.

Avaliar a funcionalidade do idoso por meio da capacidade física pode contribuir positivamente para a sua saúde. Testes físicos permitem identi- ficar precocemente a necessidade de intervenção, melhorando, assim, o prognóstico clínico. Diante disso, mostra-se relevante avaliar separadamente os fatores associados à capacidade física de membros superiores e inferiores, tendo em vista suas especificidades e consequências para a vida do idoso; bem como identificar precocemente aqueles com maior risco de desenvolver declínio funcional, iniciativa que se traduz como uma das metas da Política Nacional de Saúde da Pessoa Idosa (PNSPI) para o alcance do envelhecimento saudável ${ }^{12}$. Desse modo, o presente estudo buscou avaliar os fatores relacionados à capacidade física de membros inferiores e de membros superiores em idosos quilombolas.

\section{Metodologia}

Este é um estudo transversal, com abordagem quantitativa e coleta de dados primários, que faz parte de um estudo maior intitulado "Avaliação multidimensional da saúde de idosos de uma comunidade quilombola do estado da Paraíba".

Foram incluídos idosos (60 anos ou mais) de ambos os sexos, residentes na comunidade quilombola Caiana dos Crioulos, cadastrados no ESUS. Foram excluídos idosos com declínio cognitivo que declararam pessoalmente, ou por meio de informante, serem portadores de deficiência visual e/ou auditiva grave não corrigida; acamados ou cuja condição física o impossibilitasse de andar, ou realizar atividades de capacidade física; que estiveram ausentes do quilombo no período de coleta dos dados.

Para o rastreio de declínio cognitivo foi utilizado o Mini-Exame do Estado Mental (MEEM) ${ }^{13}$ com os seguintes pontos de corte: analfabetos (pontuação $<13$ indica quadro demencial, $\geq 13$ sem quadro demencial); Fundamental e Médio, $<8$ anos de estudo (pontuação $<18$ indica quadro demencial, $\geq 18$ sem quadro demencial); alto nível de escolaridade, $>8$ anos de estudo $(<26$ indica quadro demencial, $\geq 26$ sem quadro demencial).

\section{Coleta de dados}

Inicialmente, foi realizado um estudo-piloto na comunidade quilombola de Pedra D’água em Ingá, na Paraíba. Participaram do estudo-piloto 18 idosos, o equivalente a $32 \%$ dos idosos residentes em Caiana dos Crioulos.

A coleta dos dados dos idosos da comunidade Quilombola de Caiana dos Crioulos, no municí- 
pio de Alagoa Grande/PB, Brasil, foi realizada no período de outubro a dezembro de 2019. A cidade encontra-se localizada no interior do Estado, a $111 \mathrm{~km}$ da capital, João Pessoa. Inicialmente foi realizada uma reunião com a representante da comunidade quilombola e com a ACS responsável pela área, na qual foi exposta a metodologia e objetivos da pesquisa. Ambas foram convidadas a serem multiplicadoras da informação, auxiliando na comunicação com a comunidade.

Foi obtida uma lista de idosos da comunidade na Unidade Básica de Saúde (UBS), que permitiu o planejamento de um cronograma com dias e horários marcados para o comparecimento ao local de pesquisa. Desta maneira, os idosos foram convidados a se dirigirem à sede da associação de moradores do quilombo em dia e horário predeterminados. Para o idoso que não compareceu foi realizada uma nova chamada. Em caso de nova falta, os idosos foram visitados em seu domicílio pela equipe de pesquisadores, com a possibilidade de duas visitas. Os idosos ausentes nas duas visitas foram considerados perda.

As variáveis avaliadas foram: capacidade física de membros superiores e inferiores (variáveis dependentes), situação socioeconômico-demográfica, sintomas indicativos de depressão, situação de saúde, prática de atividade física e comportamento sedentário (variáveis independentes).

A capacidade física de membros superiores foi verificada por meio da força de preensão manual. Inicialmente, o idoso foi questionado sobre o braço que considerava ter maior força. Os idosos foram questionados, ainda, quanto à realização de cirurgias no braço ou na mão no período de três meses anteriores à coleta dos dados, em caso afirmativo estes foram excluídos do teste. A força de preensão manual foi aferida com a utilização de um dinamômetro hidráulico manual (Dinamômetro Takei Kiki Kogyo ${ }^{\circledR}$ TK 1201, Japão) ajustado para o tamanho das mãos de cada idoso. Este procedimento foi realizado três vezes, com um intervalo entre as execuções (um minuto), sendo considerada a média como valor final. Quanto maior o valor médio, melhor a capacidade física.

A capacidade dos membros inferiores foi avaliado por meio da versão brasileira da Short Physical Performance Battery (SPPB) ${ }^{3}$, que constitui instrumento eficaz para avaliar a capacidade dos membros inferiores da população idosa. É composta por uma sequência de três testes que avaliam o equilíbrio estático em pé, a velocidade de marcha e a força muscular dos membros infe- riores. A pontuação de cada teste varia de 0 (pior desempenho) a 4 pontos (melhor desempenho). A classificação da SPPB é dada pelo somatório dos escores dos três testes, que pode variar de $0 \mathrm{a}$ 12. Quanto maior o valor somado melhor a capacidade física do idoso.

Foram verificadas questões referentes à idade, anos de estudo, número de residentes no domicílio (soma das pessoas que moravam na mesma residência, incluindo o idoso), renda familiar per capita (soma da renda bruta da família dividida pelo número de pessoas que moravam no domicílio). O nível socioeconômico foi obtido a partir do questionário da Associação Brasileira de Empresas de Pesquisa, gerando uma pontuação referente a estratificação econômica: classes $\mathrm{A} / \mathrm{B}$ ( 29 a 100 pontos), C (17 a 28 pontos) e D/E (0 a 16 pontos). Valores maiores são indicativos de melhor nível socioeconômico ${ }^{14}$.

Os sintomas indicativos de depressão foram analisados por meio da Escala de Depressão Geriátrica (GDS) ${ }^{15}$ composta por 15 perguntas, com escore total que varia de 0 a 15 pontos, cada pergunta possui duas alternativas (sim, não) com valores atribuídos de 0 a 1 ponto. Uma pontuação acima de cinco pontos é indicativa de presença de sintomas indicativos de depressão.

A prática de atividade física e o comportamento sedentário foram analisados por meio do International Physical Activity Questionnaire (IPAQ) versão curta ${ }^{16}$. O questionário é constituído por perguntas referentes à frequência, duração e intensidade da prática de atividade física. Quanto maior a duração da prática de atividade física, em minutos por semana, mais ativo é o indivíduo. Usando o compêndio de atividades físicas, foi obtido a atividade física total em ME$\mathrm{T}$-min/semana. O metabolic equivalente intensity level (MET) é uma medida que estima o gasto energético com atividade física. Foram utilizadas as pontuações médias de MET para cada domínio (Caminhada= 3,3 METs; Moderada = 4,0 METs; Vigorosa $=8,0$ METs), dessa forma, foi utilizada a equação: média de MET x minutos de atividade $\mathrm{x}$ frequência por semana. Assim, foram obtidos valores contínuos em METs-minutos/semana ${ }^{17}$.

Para avaliar o comportamento sedentário foi utilizado o domínio tempo sentado durante um dia da semana e um dia no final de semana. Foi executado um cálculo de média ponderada, no qual o tempo sentado indicado para os dias da semana foi multiplicado por cinco, depois somado ao tempo dos dias de final de semana, multiplicado por dois. Este resultado foi dividido por sete, para obter o número médio de horas por dia 
despendidos na posição sentada ${ }^{18}$. O ponto de corte indicado para comportamento sedentário "sim" é de $>180$ minutos $^{19}$. Quanto maior o tempo sentado em minutos por semana, maior é a indicação de comportamento sedentário.

A situação de saúde foi avaliada por meio de informações sobre o número de morbidades referidas, ocorrência de quedas, estado nutricional e risco metabólico. O número de morbidades referidas foi obtido questionando ao idoso se algum médico ou outro profissional da saúde lhe informou que ele apresentava alguma doença das citadas: hipertensão arterial; problema cardíaco; osteoporose; derrame ou acidente vascular cerebral; artrite/ artrose ou reumatismo; diabetes mellitus; câncer; doença crônica pulmonar; glaucoma; doença de Parkinson. A ocorrência de quedas foi avaliada por meio da resposta para o seguinte questionamento: $o(a)$ senhor (a) caiu nos últimos 12 meses?, em caso afirmativo foi questionado o número de vezes.

$\mathrm{O}$ estado nutricional foi verificado por meio do Índice de Massa Corporal (IMC) (peso (kg)/ estatura $\left.(\mathrm{m})^{2}\right)$, do perímetro da panturrilha (PP) e da circunferência muscular do braço (CMB). $\mathrm{O}$ peso e a estatura foram mensurados com a utilização de balança eletrônica digital portátil (TANITA UM080 ${ }^{\circledR}$ ) e estadiômetro portátil (ALTUREXATA $^{\circledR}$ ), respectivamente ${ }^{20}$. De acordo com a classificação sugerida pela Organización Panamericana de la Salud (OPAS) ${ }^{21}$ valores de IMC $\leq 23$ $\mathrm{kg} / \mathrm{m}^{2}$ são indicativos de baixo peso, $>23 \mathrm{~kg} / \mathrm{m}^{2}$ $\mathrm{e}<28 \mathrm{~kg} / \mathrm{m}^{2}$ indicam eutrofia, $\geq 28 \mathrm{~kg} / \mathrm{m}^{2} \mathrm{e}<30$ $\mathrm{kg} / \mathrm{m}^{2}$ indicam sobrepeso e $\geq 30 \mathrm{~kg} / \mathrm{m}^{2}$ indicam obesidade. Quanto menor o IMC maior o risco de desnutrição e quanto maior o IMC maior o risco de sobrepeso/obesidade.

O PP foi aferido com fita métrica inelástica ${ }^{20}$. Os seguintes valores são indicativos de massa muscular diminuída: $\leq 34 \mathrm{~cm}$ para homens e $\leq$ $33 \mathrm{~cm}$ para mulheres ${ }^{22}$. A CMB foi obtida a partir da seguinte equação ${ }^{23}$ : $\mathrm{CMB}(\mathrm{cm})=[\mathrm{PB}(\mathrm{cm})-$ ( a dobra cutânea tricipital (DCT) foram aferidos com a utilização de fita métrica inelástica e do compasso de dobras cutâneas Lange ${ }^{\oplus}$, respectivamente $^{20}$. Valores menores de CMB são indicativos de desnutrição.

O risco metabólico foi verificado por meio da circunferência da cintura (CC), de acordo com as técnicas descritas em Lohman et al. ${ }^{20}$. A presença de risco metabólico foi verificada por meio dos seguintes valores de CC: $\geq 102 \mathrm{~cm}$ para homens e $\geq 88 \mathrm{~cm}$ para mulheres ${ }^{24}$.

\section{Procedimentos estatísticos}

Os dados foram submetidos à dupla entrada, cuja validação foi verificada por meio da ferramenta "validate" (Epi Info 7.2). Os dados descritivos são apresentados sob a forma de média, mediana, intervalos interquartílicos, desvio padrão, valor máximo e mínimo. A normalidade da distribuição dos dados foi analisada pelo teste de Anderson- Darling.

Foi realizado o teste de correlação de Spearman, assim como foram construídos modelos de regressão linear simples, considerando as variáveis independentes com relação às variáveis dependentes (nível de significância de 5\%). Para testar a relação conjunta das variáveis independentes sobre a FPM e a pontuação da SPPB construiu-se um modelo inicial de regressão linear múltipla para cada variável dependente. As variáveis com $p$ valor menor que 0,20 em cada modelo inicial foram incluídas em um modelo final de regressão linear múltipla. Permaneceram no modelo final as variáveis que apresentaram nível de significância de $p<0,05$. Para a regressão linear múltipla utilizou-se o método de Backward. $\mathrm{Na}$ sequência por etapas (Stepwise), foram retiradas as variáveis que apresentavam maior probabilidade de não ter relação com as variáveis dependentes (FPM e a SPPB). As análises foram realizadas com o auxílio do software estatístico R ( $\mathrm{R}$ CORE TEAM, 2019).

\section{Aspectos éticos}

A pesquisa maior da qual este estudo faz parte, foi aprovada pelo Comitê de Ética em Pesquisa (CEP) da Universidade Estadual da Paraíba. Os idosos, ao aceitarem participar foram orientados a assinarem o Termo de Consentimento Livre e Esclarecido, conforme preconiza a Resolução ${ }^{\circ}$ 466/2012 do Conselho Nacional de Saúde do Ministério da Saúde.

\section{Resultados}

Esse estudo teve a participação de 43 idosos quilombolas, com média etária entre 70 e 74 anos $( \pm 6,66)$, variando de 60 a 83 anos. Do total de idosos residentes na comunidade quilombola (55), quatro recusaram participar da pesquisa, dois não foram encontrados em seus domicílios nas duas visitas realizadas, dois foram excluídos por não conseguirem responder aos questionários, três foram excluídos segundo o critério do 
MEEM, um idoso foi excluído por não conseguir executar os testes físicos.

Na Tabela 1 são apresentados os valores referentes à média, desvio-padrão, mediana, valor máximo, valor mínimo, primeiro quartil e terceiro quartil das variáveis estudadas. A média dos valores da força de preensão manual foi 22,44 kgf e da pontuação da Short Physical Performance Battery (SPPB) foi 8,47 $( \pm 2,09)$.

Na Tabela 2 são apresentados os resultados da análise de regressão linear simples via modelo bivariado, entre a força de preensão manual e as variáveis independentes avaliadas. É possível observar que houve correlação positiva estatisticamente significativa entre FPM e PAF $\left(\mathrm{R}^{2}=0,141\right.$; $\mathrm{p}=0,013)$, mostrando que a cada unidade de aumento da PAF (METs-min/sem) ocorre aumento de 0,000048 de unidade do valor da FPM (kgf) $\left(\beta_{1}=0,000048\right)$. Houve correlação positiva estatisticamente significativa entre FPM e CMB $\left(\mathrm{R}^{2}=0,237 ; \mathrm{p}=0,0009\right)$, assim a cada unidade de aumento no valor da CMB $(\mathrm{cm})$ ocorre aumento de 1,199 de unidade no valor da FPM (kgf) $\left(\beta_{1}=1,199\right)$.

Na Tabela 3 são apresentados os resultados da análise de regressão linear simples via mode- lo bivariado, entre a pontuação da Short Physical Performance Battery (SPPB) e as variáveis independentes. É possível observar que houve correlação inversa entre a pontuação da SPPB e a idade $\left(R^{2}=0,236 ; p=0,0009\right)$. Sendo assim, de acordo com o modelo ajustado dos dados, a cada unidade de aumento na idade ocorre diminuição de 0,152 de unidade de pontuação da SPPB $\left(\beta_{1}=\right.$ $-0,152)$.

Na Tabela 4 são apresentados os resultados da regressão linear múltipla entre as variáveis independentes e a FPM e a SPPB. A idade, a CMB e os anos de estudo apresentaram correlação significativa com a FPM $(\mathrm{p}<0,05)$, explicando $37 \%$ $\left(\mathrm{R}^{2}=0,374\right)$ da variação da FPM entre os idosos quilombolas. A CMB apresentou efeito significativo diretamente proporcional $(\beta 1=1,305)$ com a FPM, ou seja, cada unidade de aumento de CMB corresponde a um aumento de 1,305 na unidade do valor da FPM. A idade e anos de estudo apresentaram efeito significativo inversamente proporcional $(\beta 1=-0,373$ e $\beta 1=-2,726$, respectivamente) com a FPM, ou seja, cada unidade de aumento na idade e nos anos de estudo corresponde a uma diminuição de 0,373 e 2,726 , respectivamente, na unidade do valor da FPM.

Tabela 1. Média, desvio-padrão, mediana, valor mínimo, valor máximo, $1^{\circ}$ quartil e $3^{\circ}$ quartil das variáveis relativas à capacidade física de membros superiores e inferiores, situação socioeconômico-demográfica, sintomas indicativos de depressão, situação de saúde, prática de atividade física e comportamento sedentário. Alagoa Grande, Brasil, 2019.

\begin{tabular}{lrrrrrrr}
\hline \multicolumn{1}{c}{ Variáveis } & Média & $\begin{array}{c}\text { Desvio } \\
\text { Padrão }\end{array}$ & Mediana & Máximo & Mínimo & $\begin{array}{c}\mathbf{1}^{\mathbf{0}} \\
\text { Quartil }\end{array}$ & $\begin{array}{c}\mathbf{3}^{\mathbf{2}} \\
\text { Quartil }\end{array}$ \\
\hline FPM (kgf) & 22,44 & 8,93 & 22,7 & 47,3 & 7,6 & 15,8 & 27,2 \\
SPPB (pontos) & 8,47 & 2,09 & 9 & 12 & 4 & 7 & 12 \\
Idade (anos) & 70,74 & 6,66 & 70 & 83 & 60 & 65,5 & 75 \\
Nível socioeconômico (pontos) & 12,6 & 2,8 & 12 & 22 & 7 & 11 & 14 \\
Renda per capita familiar (R\$) & 868,899 & 520,21 & 748,5 & 1.996 & 166,3 & 487 & 998 \\
Número de residentes no domicílio & 2,84 & 1,56 & 2 & 7 & 1 & 2 & 4 \\
Anos de estudo & 0,65 & 1 & 0 & 4 & 0 & 0 & 1 \\
GDS15 (pontos) & 3,42 & 2,34 & 3 & 11 & 1 & 2 & 4 \\
PAF (METs-min/sem) & $5.725,09$ & $6.986,98$ & 2.388 & 27.540 & 0 & 873 & $8.418,5$ \\
Comportamento sedentário (min/sem) & 207,31 & 145,42 & 197,14 & 720 & 30 & 111,42 & 300 \\
Número de morbidades referidas & 1,54 & 1,25 & 1 & 5 & 0 & 1 & 2 \\
Número de quedas & 0,44 & 0,73 & 0 & 2 & 0 & 0 & 1 \\
IMC (kg/m ${ }^{2}$ ) & 26,92 & 4,87 & 26,98 & 37,38 & 17,17 & 23,38 & 30,41 \\
CMB (cm) & 22,39 & 3,63 & 21,98 & 31,13 & 15,68 & 19,23 & 25,62 \\
PP (cm) & 33,41 & 4,22 & 33,9 & 40,9 & 21,6 & 30,65 & 36,5 \\
CC (cm) & 92,71 & 12,73 & 93 & 121,8 & 62,46 & 85 & 101,9 \\
\hline
\end{tabular}

FPM: Força de preensão manual; SPPB: Short Physical Performance Battery; GDS: Geriatric Depression Scale; PAF: Prática de atividade física; IMC: Índice de Massa Corporal; CMB: Circunferência muscular do braço; PP: Perímetro da panturrilha; CC: Circunferência da cintura. 
Tabela 2. Ajuste do modelo de regressão linear simples via modelo bivariado entre FPM e variáveis independentes em idosos quilombolas, Alagoa Grande. Paraíba, Brasil. 2019.

\begin{tabular}{|c|c|c|c|c|c|c|}
\hline \multirow[b]{2}{*}{ Variáveis } & \multicolumn{6}{|c|}{ Força de preensão manual (FPM) } \\
\hline & r (IC 95\%) & $\begin{array}{c}\text { p- } \\
\text { valor }\end{array}$ & $\begin{array}{c}\text { Estimativa } \beta 0 \\
(p \text {-valor })\end{array}$ & $\begin{array}{c}\text { Estimativa } \\
\beta 1\end{array}$ & $\mathbf{R}^{2}$ & $\begin{array}{c}\text { p- } \\
\text { valor }\end{array}$ \\
\hline Idade & $-0,179(-0,452-0,136)$ & 0,250 & $-40,600(0,008)^{\star}$ & $-0,256$ & 0,036 & 0,218 \\
\hline $\begin{array}{l}\text { Nível socioeconômico } \\
\text { (pontos) }\end{array}$ & $0,154(-0,136-0,439)$ & 0,322 & $15,330(0,019)^{\star}$ & 0,564 & 0,031 & 0,256 \\
\hline $\begin{array}{l}\text { Renda per capita familiar } \\
(\mathrm{R} \$)\end{array}$ & $-0,192(-0,500-0,126)$ & 0,216 & $25,468(<0,0001)$ & $-0,003$ & 0,041 & 0,192 \\
\hline Anos de estudo & $-0,250(-0,507-0,044)$ & 0,104 & $23,621(<0,0001)$ & $-1,815$ & 0,041 & 0,193 \\
\hline $\begin{array}{l}\text { Número de residentes no } \\
\text { domicílio }\end{array}$ & $0,114(-0,198-0,440)$ & 0,465 & $20,482(<0,0001)$ & 0,689 & 0,014 & 0,442 \\
\hline GDS15 (pontos) & $-0,285(-0,551-0,017)$ & 0,063 & $25,437(<0,0001)$ & $-0,877$ & 0,052 & 0,138 \\
\hline PAF (METs-min/sem) & $0,212(-0,132-0,490)$ & 0,170 & $19,69(<0,0001)$ & 0,0004 & 0,141 & $0,013^{\star}$ \\
\hline Comp. sedentário (min/sem) & $0,071(-0,284-0,384)$ & 0,648 & $20,465(<0,0001)$ & 0,009 & 0,024 & 0,321 \\
\hline $\begin{array}{l}\text { Número de morbidades } \\
\text { referidas }\end{array}$ & $-0,087(-0,394-0,232)$ & 0,586 & $23,664(<0,0001)$ & $-0,765$ & 0,010 & 0,516 \\
\hline Número de quedas & $-0,173(-0,438-0,112)$ & 0,265 & $23,572(<0,0001)$ & $-2,564$ & 0,044 & 0,175 \\
\hline $\operatorname{IMC}\left(k g / m^{2}\right)$ & $0,099(-0,214-0,371)$ & 0,527 & $17,632(0,029)^{\star}$ & 0,178 & 0,009 & 0,534 \\
\hline $\mathrm{CMB}(\mathrm{cm})$ & $0,478(0,222-0,680)$ & $0,001^{\star}$ & $-4,400(0,565)$ & 1,199 & 0,237 & $0,0009^{*}$ \\
\hline $\mathrm{PP}(\mathrm{cm})$ & $0,199(-0,108-0,498)$ & 0,198 & $4,259 \quad(0,694)$ & 0,544 & 0,065 & 0,096 \\
\hline $\mathrm{CC}(\mathrm{cm})$ & $0,077(-0,264-0,381)$ & 0,620 & $12,592(0,221)$ & 0,106 & 0,022 & 0,333 \\
\hline
\end{tabular}

FPM: Força de preensão manual; SPPB: Short Physical Performance Battery; GDS: Geriatric Depression Scale; PAF: Prática de atividade física; IMC: Índice de Massa Corporal; CMB: Circunferência muscular do braço; PP: Perímetro da panturrilha; CC: Circunferência da cintura; r: coeficiente de correlação; ${ }^{\star}$ Nível de significância $<0,05 ; \beta 0$ : intercepto; $\beta 1$ : coeficiente angular; R $^{2}$ : coeficiente de determinação.

Fonte: Dados da pesquisa, 2019.

Quanto à pontuação da SPPB, a idade é a variável significativa $(\mathrm{p}<0,05)$ que compõe o modelo final, a qual explica $25 \%\left(\mathrm{R}^{2}=0,2524\right)$ da variação da SPPB entre os idosos quilombolas. A idade apresentou efeito significativo inversamente proporcional $(\beta 1=-0,15003)$ à SPPB. Cada unidade de aumento na idade corresponde a uma diminuição de 0,15003 de unidade da pontuação da SPPB.

\section{Discussão}

A capacidade física retrata a habilidade que o indivíduo apresenta para desenvolver uma atividade de forma independente e reflete o estado de funcionalidade. Diversos fatores estão associados à capacidade física como a flexibilidade, força, equilíbrio e condicionamento aeróbico ${ }^{25}$. Santos et al. ${ }^{2}$ expõem que a avaliação desses fatores em idosos é imprescindível para o planejamento de intervenções mais adequadas, que atendam as demandas expostas por esse grupo. A implementação de ações voltadas para os idosos é prevista nas diretrizes do Ministério da Saúde, todavia ainda é insipiente nos serviços de atenção primária, principalmente para as atividades voltadas para o envelhecimento saudável, o que chama atenção para a urgência de melhorias no desempenho dos serviços do SUS ${ }^{26}$.

É possível que este seja o primeiro estudo que objetivou avaliar os fatores associados à capacidade física de membros superiores e inferiores em idosos quilombolas. Os quilombolas por constituírem uma população vulnerável, muitas vezes são tratados pelas políticas públicas de uma forma macro, ou seja, no mesmo nível que os demais grupos vulneráveis. No entanto, é preciso reconhecer as especificidades de cada um dos grupos, desta população vulnerável. Embora tenhamos políticas para idosos ${ }^{12}$ e para negros ${ }^{27}$; e agenda social para quilombolas ${ }^{28}$; não há conhecimento de uma política pública ou agenda específica para idosos quilombolas, tornando este grupo invisível, por conseguinte suas necessidades.

Os achados deste estudo indicaram relação entre a capacidade física de membros superiores e a idade, a CMB e os anos de estudo; assim como, a relação entre a capacidade física de 
Tabela 3. Ajuste do modelo de regressão linear simples via modelo bivariado entre SPPB e variáveis independentes em idosos quilombolas. Alagoa Grande, Paraíba, Brasil. 2019.

\begin{tabular}{|c|c|c|c|c|c|c|}
\hline \multirow[b]{2}{*}{ Variáveis } & \multicolumn{6}{|c|}{ Short Physical Performance Battery (SPPB) } \\
\hline & r (IC 95\%) & $\begin{array}{c}\text { p- } \\
\text { valor }\end{array}$ & $\begin{array}{c}\text { Estimativa } \beta 0 \\
(p-v a l o r)\end{array}$ & $\begin{array}{c}\text { Estimativa } \\
\beta 1 \\
\end{array}$ & $\mathbf{R}^{2}$ & $\begin{array}{c}\text { p- } \\
\text { valor }\end{array}$ \\
\hline Idade & $-0,467(-0,675-0,209)$ & $0,001^{*}$ & $19,238(<0,0001)$ & $-0,152$ & 0,236 & $0,0009^{*}$ \\
\hline $\begin{array}{l}\text { Nível socioeconômico } \\
\text { (pontos) }\end{array}$ & $-0,144(-0,427-0,167)$ & 0,354 & $10,262(<0,0001)$ & $-0,142$ & 0,036 & 0,218 \\
\hline $\begin{array}{l}\text { Renda per capita familiar } \\
(\mathrm{R} \$)\end{array}$ & $-0,302(-0,562-0,014)$ & $0,048^{*}$ & $9,132(<0,0001)$ & $-0,000$ & 0,036 & 0,218 \\
\hline Anos de estudo & $0,119(-0,191-0,379)$ & 0,445 & $8,169(<0,0001)$ & 0,454 & 0,047 & 0,162 \\
\hline $\begin{array}{l}\text { Número de residentes no } \\
\text { domicílio }\end{array}$ & $0,207(-0,098-0,469)$ & 0,180 & $7,817(<0,0001)$ & 0,228 & 0,029 & 0,274 \\
\hline GDS15 (pontos) & $-0,142(0,424-0,158)$ & 0,360 & $8,737(<0,0001)$ & $-0,079$ & 0,008 & 0,568 \\
\hline PAF (METs-min/sem) & $0,318(-0,0364-0,589)$ & $0,037^{\star}$ & $7,962(<0,0001)$ & 0,000 & 0,086 & 0,055 \\
\hline $\begin{array}{l}\text { Comp. sedentário ( } \mathrm{min} / \\
\text { sem) }\end{array}$ & $-0,195(-0,5006-0,123)$ & 0,208 & $8,901(<0,0001)$ & $-0,002$ & 0,021 & 0,348 \\
\hline $\begin{array}{l}\text { Número de morbidades } \\
\text { referidas }\end{array}$ & $-0,172(-0,4906-0,184)$ & 0,281 & $9,098(<0,0001)$ & $-0,397$ & 0,054 & 0,142 \\
\hline Número de quedas & $-0,076(-0,3702-0,213)$ & 0,626 & $8,461(<0,0001)$ & 0,007 & 0,000 & 0,987 \\
\hline $\operatorname{IMC}\left(k g / m^{2}\right)$ & $0,066(-0,235-0,3604)$ & 0,670 & $7,349(0,0002)^{\star}$ & 0,041 & 0,009 & 0,536 \\
\hline $\mathrm{CMB}(\mathrm{cm})$ & $0,085(-0,205-0,378)$ & 0,584 & $7,890(0,0003)^{\star}$ & 0,025 & 0,002 & 0,775 \\
\hline $\mathrm{PP}(\mathrm{cm})$ & $0,128(-0,2102-0,419)$ & 0,412 & $5,178(0,048)^{\star}$ & 0,098 & 0,039 & 0,201 \\
\hline $\mathrm{CC}(\mathrm{cm})$ & $-0,010(-0,303-0,294)$ & 0,947 & $8,402(0,001)^{\star}$ & 0,0006 & 0,00001 & 0,979 \\
\hline
\end{tabular}

FPM: Força de preensão manual; SPPB: Short Physical Performance Battery; GDS: Geriatric Depression Scale; PAF: Prática de atividade física; IMC: Índice de Massa Corporal; CMB: Circunferência muscular do braço; PP: Perímetro da panturrilha; CC: Circunferência da cintura; r: coeficiente de correlação; ${ }^{*}$ Nível de significância $<0,05 ; \beta 0$ : intercepto; $\beta 1$ : coeficiente angular; $R^{2}$ : coeficiente de determinação.

Fonte: Dados da pesquisa, 2019.

Tabela 4. Ajuste de regressão linear múltipla via modelo bivariado para estimar a predição das variáveis independentes sobre a FPM e a pontuação da SPPB em idosos quilombolas. Alagoa Grande, Paraíba, Brasil. 2019.

\begin{tabular}{|c|c|c|c|c|c|c|}
\hline & Variáveis & $\mathbf{R}^{2}$ & $\beta 1$ & Erro padrão & Estatística t & p-valor \\
\hline Força de & & 0,374 & & & & \\
\hline Preensão & Intercepto & & 21,376 & 13,8408 & 1,544 & 0,1305 \\
\hline \multirow{3}{*}{$\begin{array}{l}\text { Manual } \\
\text { (FPM) }\end{array}$} & Idade & & $-0,373$ & 0,173 & $-2,15$ & $0,037^{\star}$ \\
\hline & $\mathrm{CMB}(\mathrm{cm})$ & & 1,305 & 0,313 & 4,163 & $0,0001^{*}$ \\
\hline & Anos de estudo & & $-2,726$ & 1,162 & $-2,346$ & $0,024^{*}$ \\
\hline \multirow{5}{*}{$\begin{array}{l}\text { Short Physical } \\
\text { Performance } \\
\text { Battery } \\
\text { (SPPB) }\end{array}$} & & 0,2524 & & & & \\
\hline & Intercepto & & 19,918 & 3,176 & 6,270 & $<0,0001$ \\
\hline & Idade & & $-0,15003$ & 0,044 & $-3,354$ & $0,00178^{*}$ \\
\hline & Nível socioeconômico (pontos) & & $-0,074$ & 0,104 & $-0,706$ & 0,484 \\
\hline & Número de quedas & & 0,213 & 0,398 & 0,536 & 0,595 \\
\hline
\end{tabular}

FPM: Força de preensão manual; SPPB: Short Physical Performance Battery; CMB: Circunferência muscular do braço; * Nível de significância $<0,05 ; \beta 0$ : intercepto; $\beta 1$ : coeficiente angular; $R^{2}$ : coeficiente de determinação.

Fonte: Dados da pesquisa, 2019.

membros inferiores e a idade. Informações como estas podem auxiliar os profissionais da saúde para adoção de medidas de prevenção específicas para a população, contribuindo para a promoção da saúde com estímulo das habilidades físicas, favorecendo positivamente a capacidade física e funcional, com consequente efeito na proteção da saúde e na longevidade, assim como di- 
minuindo gastos futuros com serviços de maior complexidade.

$\mathrm{Na}$ análise bivariada deste estudo, observouse correlação significativa entre FPM e CMB, e entre FPM e prática de atividade física. O modelo de regressão múltipla identificou três fatores (a idade, a CMB e os anos de estudo) que, em conjunto, explicam $37 \%$ da variabilidade da capacidade física de membros superiores. A correlação entre FPM e CMB, corrobora com resultados obtidos em outros estudos realizados com idosos ${ }^{29,30}$. Silva et al. ${ }^{29}$ avaliando 420 idosos de ambos os sexos, encontraram correlação positiva e significativa entre FPM e CMB. Os autores discutem que variações nos indicadores antropométricos podem interferir na FPM desses idosos. Estudo realizado na cidade de São Caetano do Sul, São Paulo observou associação entre menores valores de CMB e FPM diminuída, o que segundo os autores, confirma a CMB como um indicador sensível à redução muscular ${ }^{30}$.

A CMB apresenta-se como indicador de reserva muscular, que durante o envelhecimento é comum reduzir. Menor reserva muscular se relaciona com menor força muscular ${ }^{29}$. Essa redução da massa muscular pode resultar em déficit em capacidades físicas e, consequentemente, influenciar para o aparecimento de incapacidades e fragilidade. Assim, observa-se a importância de avaliar o comportamento deste importante indicador sobre diferentes medidas de capacidades físicas. Aspecto abordado por este estudo de forma particular.

Quanto à correlação inversa entre FPM e idade, verificada neste estudo, resultado semelhante foi encontrado por Lima et al. ${ }^{31} \mathrm{em}$ estudo realizado com adultos e idosos, o qual verificou que a idade foi fator associado a menores valores de FPM. De acordo com os autores, essa relação pode ser justificada pelo enfraquecimento da musculatura com o passar dos anos, pela diminuição da massa muscular; além fato de os idosos mais velhos do estudo serem mais inativos. Marques et al. ${ }^{30}$ encontraram associação entre a FPM diminuída e a maior idade. A literatura apresenta clara relação inversa entre a idade e a FPM. Essa perda está associada principalmente à hipotrofia muscular resultante do processo de sarcopenia, além dos baixos níveis de prática de atividade física entre os idosos, que contribui para o desuso da musculatura e consequente dinapenia nessa população.

A relação inversa entre FPM e anos de estudos observada neste estudo mostra que quando menor o número de anos de estudos maior o valor da FPM, por conseguinte, melhor a capacidade física. Essa relação inversa pode estar relacionada ao fato de que indivíduos com baixa escolaridade estariam mais propensos a trabalhos que exigem maior esforço que, consequentemente, auxiliariam na melhora da força. De acordo com Miranda et al. ${ }^{9}$ a convergência de raça/etnia, gênero, região, classe e escolaridade contribuem para a exploração da força de trabalho, precariedade e manutenção das iniquidades sociais. Os quilombolas desenvolvem principalmente trabalhos ligados à agricultura e à construção civil. A informalidade não tem garantia de direitos trabalhistas, recolhimento de contribuição ou benefícios para os trabalhadores. Neste sentido, políticas públicas focadas nos quilombolas apresentam-se como linhas de fuga e necessitam de articulação com o Estado, além da participação de todos os envolvidos.

Apesar desta relação inversa, há que se considerar a importância da educação como meio para promover a saúde. Ribeiro et al. ${ }^{32}$ verificaram em estudo a influência da educação sobre a saúde, mostrando repercussão positiva na condição socioeconômica, que proporcionou mudanças no estilo de vida. Assim, a escolarização atuou como forma promotora de saúde, com a adoção de hábitos saudáveis, prática de atividade física, melhoria no entendimento das orientações em saúde. Logo, tendo em vista que a educação é promotora de saúde deve ser proporcionada e incentivada para toda a população.

O valor médio da SPPB verificado neste estudo foi semelhante ao encontrado no primeiro momento de um estudo longitudinal de dois anos com idosos, que encontrou escore médio de $8,46( \pm 2,68)$ pontos $^{33}$. Este valor médio é indicativo de moderada capacidade física de membros inferiores, que pode estar relacionado ao elevado comportamento sedentário apesar dos idosos apresentarem prática de atividade física adequada.

Diante disso, Pavasini et al. ${ }^{34}$ verificaram em uma revisão sistemática e meta-análise que escore da SPPB menor que dez pontos é indicativo de mortalidade por todas as causas. Dessa forma, é importante considerar o declínio da pontuação deste instrumento, uma vez que consiste em declínio da capacidade física, indicando necessidade de intervenção nesta população. Mesmo que o valor médio apresentado neste estudo seja sugestivo de capacidade moderada, torna-se necessário que os idosos sejam monitorados, tendo em vista a importância desta variável para a funcionalidade do idoso. 
Neste estudo, tanto na análise bivariada como na múltipla, a idade esteve relacionada de forma inversa com a SPPB. No modelo múltiplo, apenas a idade apresentou correlação com a SPPB, explicando variabilidade de $25 \%$ da capacidade física de membros inferiores. Resultado semelhante foi verificado em outros estudos ${ }^{35,36}$. Ikegami et al. ${ }^{33}$ observaram relação entre faixa etária e a SPPB, na qual idosos com idades mais avançadas apresentam piores pontuações. Os autores discutem que embora a idade cronológica não seja um marcador preciso para as alterações do envelhecimento, o passar dos anos gera decréscimos na função física, aspecto esse que acarreta prejuízos de mobilidade, equilíbrio e força.

De forma ampla, o progredir da idade atua sobre diversos aspectos do indivíduo. Silva et al. ${ }^{37}$ discutem que, nos idosos, ocorre redução do número de unidades motoras, redução de fibras musculares, diminuição da eficiência do sistema neurológico, aumento da fraqueza muscular e fadiga, lentidão nos movimentos, que contribuem para as limitações para caminhar, levantar-se e executar atividades.

Essas alterações relacionadas à idade justificam os achados deste estudo em que a idade se mostrou inversamente relacionada tanto à FPM como à SPPB no modelo múltiplo. Dessa forma, quanto maior a idade pior a capacidade física tanto de membros superiores como de membros inferiores. A que se considerar que envelhecer com saúde exige estratégias de promoção da saúde em todas as idades, desde o nascimento até a velhice; sendo assim, ações de prevenção devem ser direcionadas a todos os grupos. Para isso a PNSPI prevê o investimento na promoção da saúde em todas as idades, com a propagação de informações adequadas sobre envelhecimento a toda a população, inclusão nos currículos escolares de disciplinas que abordem o processo de envelhecimento e medidas de promoção e prevenção ${ }^{12}$.

No que se refere à avaliação das capacidades físicas, os testes utilizados nesta pesquisa apresentam características diferentes relacionadas ao recrutamento muscular. A FPM, avaliada pela dinamometria, solicita mais as fibras musculares do tipo I. O teste de sentar e levantar, incluído na SPPB, recruta em sua maioria fibras do tipo II, que são mais predominantes nos membros inferiores e sofrem maior hipotrofia ${ }^{2}$. Com isso, ressalta-se a necessidade de avaliação específica dos diferentes segmentos corporais, buscando, assim, uma avaliação mais criteriosa a respeito da capacidade física durante o processo de envelhe- cimento, para a prevenção dos desfechos decorrentes desse período da vida.

Neste estudo, a prática de atividade física mostrou-se relacionada à FPM na análise bivariada. No entanto, no modelo múltiplo perdeu a significância estatística, mostrando que, de forma conjunta, outras variáveis apresentaram maior relação com a FPM. Apesar disso, a atividade física é uma importante medida a ser incentivada, por seu impacto positivo sob a força e a massa muscular.

Os exercícios multicomponentes podem auxiliar no ganho de outras capacidades como coordenação, equilíbrio, mobilidade, que contribuem para a independência do idoso. Lenardt et al. ${ }^{38}$ sugerem que a prática de atividade física é uma estratégia preventiva de agravos que geram a fragilidade física, dessa forma proporciona benefícios diretos e indiretos aos idosos, como a melhora dos níveis de força muscular.

Torna-se relevante observar um aspecto considerado por Bezerra et al. ${ }^{39}$ que identificaram em estudo que idosos quilombolas apresentaram perfil de atividade física semelhante a de grupos rurais e que são mais ativos no trabalho e pouco ativos no lazer. Segundo os autores, esse achado pode estar relacionado à escolaridade, uma vez que a melhor escolaridade pode proporcionar melhor acesso aos recursos de saúde e oportunidades que facilitem a adoção de hábitos saudáveis. Dessa forma, sugere-se que o estabelecimento de parcerias para implementação de programas de atividades físicas e recreativas destinados a pessoa idosa, previsto no item desenvolvimento urbano da PNSPI ${ }^{12}$ alcance também as populações quilombolas.

Este trabalho apresenta algumas limitações relacionadas ao quantitativo, que embora seja limitado, a partir da descrição detalhada do cenário e da consistência dos dados, é possível fazer inferências válidas e, considerar que em condições similares a transferibilidade dos resultados é possível em populações quilombolas. Sugerimos para além de reconhecer e normatizar as diferenças culturais, que as políticas públicas sociais ao identificarem sujeitos remanescentes deste grupo vulnerável, reconhecendo as tradicionais práticas/costumes e as demandas, garantam efetivamente a execução e monitoramento de ações de saúde preventivas e clínico assistencial. Afinal, identificamos lacunas na descrição objetiva de ações na agenda social do Programa Brasil Quilombola e, nas políticas nacionais: de promoção da saúde, de Saúde Integral da População Negra, de saúde da pessoa idosa. 
Este estudo inclui-se entre os poucos realizados em agrupamentos quilombolas rurais, mesmo diante da complexa logística de coleta e visita domiciliar. As informações geradas poderão auxiliar na elaboração de ações para prevenção e manutenção da funcionalidade de idosos quilombolas, objetivando proporcionar a essa população autonomia e independência. Acredita-se que os resultados poderão apoiar ações em outras comunidades quilombolas rurais, tendo em vista a representatividade da comunidade quilombola de Caiana dos Crioulos na região.

\section{Conclusão}

Este estudo mostrou que a reserva de massa muscular, os anos de estudo e a idade foram fatores relacionados à capacidade física entre os idosos quilombolas. Quanto maior a CMB, melhor a capacidade de membros superiores. Quanto maior os anos de estudo, pior a capacidade de membros superiores. Quanto maior a idade, pior a capacidade de membros superiores e inferiores.

$\mathrm{O}$ conhecimento dos fatores relacionados à capacidade física pode contribuir com a formulação de estratégias de promoção da saúde, que visem melhorar as capacidades físicas e possibilitar melhor funcionalidade e independência para os idosos. Assim, sugere-se avaliações periódicas da CMB como indicador precoce de desnutri- ção, que pode comprometer a capacidade física, e o envolvimento em atividades funcionais. Além disso, é importante adotar medidas de prevenção e promoção da saúde em todas as idades, com o fortalecimento de ações educativas sobre o envelhecimento saudável para toda a população e de maneira assertiva nos currículos escolares.

A observância criteriosa destes aspectos e a intervenção precoce podem preservar a capacidade física e garantir a inclusão produtiva e a autonomia econômica tão pleiteada na agenda social das comunidades.

Os instrumentos utilizados neste estudo podem ser usados nos serviços de saúde para identificarem idosos com redução de capacidades físicas e fatores relacionados. Os testes poderão fornecer importantes informações sobre a funcionalidade, são de simples realização, por isso podem ser incluídos nas avaliações no âmbito da atenção primária. Entretanto, o dinamômetro hidráulico não é um instrumento disponível nas UBS, o que dificulta a utilização desse teste em específico na Atenção Básica. Assim, sugere-se estudos para validação de outros instrumentos mais acessíveis para avaliação de membros superiores. Em contrapartida, a SPPB é uma ferramenta viável para implementação nesse contexto. Diante da possibilidade desta avaliação, os idosos que apresentarem capacidade física inadequada deverão ser monitorados.

\section{Colaboradores}

BNS Furtado participou da coleta dos dados, da revisão bibliográfica, da interpretação e discussão dos dados, da redação, revisão e aprovação do artigo. RA Olinda participou da análise estatística, da interpretação dos dados e da redação do artigo. GMC Costa participou da revisão bibliográfica, da interpretação e discussão dos dados, da redação, revisão e aprovação do artigo. TN Menezes participou da concepção e delineamento do estudo, da interpretação e discussão dos dados, da redação, revisão e aprovação do artigo. 


\section{Referências}

1. Machado FN, Machado AN, Soares SM. Comparação entre a capacidade e desempenho: um estudo sobre a funcionalidade de idosos dependentes. Rev Lat Am de Enfermagem 2013;21(6):1321-1329.

2. Santos FC, Boggio EB, Souza CM, Rosa PV, Silva MF, Rosa LHT. A associação entre capacidade física e probabilidade de internação hospitalar em idosos que vivem na comunidade. Fisioter Pesqui 2017;24(3):238244.

3. Nakano MM. Versão brasileira da Short Physical Performance Battery SPPB : adaptação cultural e estudo da confiabilidade [dissertação]. Campinas (SP): Universidade Estadual de Campinas; 2007

4. Alonso AC, Ribeiro SM, Luna NMS, Peterson MD, Bocalini DS, Serra MM, Brech GC, Greve JM, Garcez-Leme LE. Association between handgrip strength, balance, and knee flexion/extension strength in older adults. Sergi G, organizador. PLoS One 2018; 13(6): 0198185.

5. Moraes EN de, Marino MC de A, Santos RR. Principais Síndromes Geriátricas. Rev Med Minas Gerais 2009; 20(1):1-11.

6. Santos GLA, Santana RF, Broca PV. Execution capacity of instrumental activities of daily living in elderly: Ethnonursing. Escola Anna Nery Rev Enfermagem 2016; 20(3):1-7.

7. Santos VC, Boery EN, Pereira R, Rosa DOS, Vilela ABA, Anjos KF, Boery RNSO. Condições Socioeconômicas e de Saúde associadas à Qualidade de vida de idosos quilombolas. Texto Context Enferm 2016; 25(2):1-9.

8. Oliveira SKM, Pereira MM, Guimarães ALS, Caldeira AP. Autopercepção de saúde em quilombolas do norte de Minas Gerais, Brasil. Cien Saude Colet 2015; 20(9):2879-90.

9. Miranda S, Oliveira J, Sampaio C, Rodrigues Neto J. Artigos Cartografia das condições de trabalho de a informalidade e a saúde mental. Interface (Botucatu) 2021; 25(e200478):1-20.

10. Cunha BS, Souza CRG, Prudente LOB, Osório NB Neto LSS. Sarcopenia em idosas quilombolas : Análise das variáveis antropométricas e de força de preensão manual. Rev Patol do Tocantins 2017; 4(3):9.

11. Silva Neto LS, Rezende FAC, Nunes DP, Maciel EDS, Travassos A, Osório NB. Associação de força de preensão palmar e osteoporose avaliada por densitometria óssea (DXA) em idosos quilombolas. Acta Fisiátrica 2018; 25(4).

12. Brasil. Ministério da Saúde (MS). Portaria $n^{\circ} 2528$ de 19 de outubro de 2006. Aprova a Política Nacional de Saúde da Pessoa Idosa. Brasília: MS; 2006.

13. Bertolucci PHF, Brucki SMD, Campacci SR, Juliano Y. O Mini-Exame do Estado Mental em uma população geral: impacto da escolaridade. Arquivos de Neuro-Psiquiatria 1994; 52(1):1-7

14. Associação Brasileira de Empresas de Pesquisa (ABEP). Critério de Classificação Econômica Brasil [Internet]. São Paulo: ABEP; 2019 [acessado em 2020 Fev 10]. Disponível em: http://www.abep.org/criterio -brasil
15. Almeida OP, Almeida SA. Short versions of the Geriatric Depression Scale: A study of their validity for the diagnosis of a major depressive episode according to ICD-10 and DSM-IV. Int J Geriatr Psychiatry 1999; 14:858-865.

16. Matsudo SM, Matdsudo VR, Araújo T, Andrade D, Andrade E, Oliveira L, Braggion G. Nível de atividade física da população do Estado de São Paulo: análise de acordo com o gênero, idade, nível socioeconômico, distribuição geográfica e de conhecimento. Rev Bras Cienc Mov 2002; 10(4):41-50.

17. Ainsworth BE, Haskell WL, Whitt MC, Irwin ML, Swartz AM, Strath SJ, O’Brien WL, Basset DR, Shimitz KH, Emplaincourt PO, Jocobs DR, Lenon AS. Compendium of Physical Activities: an update of activity codes and MET intensities. Med Sci Sport Exerc 2000 Set; 32(9):498-516.

18. Martins MO, Cavalcante VLF, Holanda GS, Oliveira CG, Maia FES, Meneses Júnior JR. Associação entre comportamento sedentário e fatores psicossociais e ambientais em adolescentes da Região Nordeste do Brasil. Rev Bras Ativ Fis Saude 2012; 17(2):143-150.

19. Rezende LFM, Sá TH, Mielke GI, Viscondi JYK, ReyLópez JP, Garcia LMT. All-Cause Mortality Attributable to Sitting Time. Am J Prev Med 2016; 51(2):253263.

20. Lohman T, Roche A, Martorell R. Anthropometric standardization reference manual. $1^{\text {a }}$ ed. Champaign, Illionois: Human Kinetics Books; 1988.

21. Organización Panamericana de la Salud (OPAS). Encuesta Multicéntrica. Salud Bienestar y envejecimento (SABE) en América Latina y el Caribe. Screening. Kingston, Jamaica: OPAS; 2001.22 p.

22. Pagotto V, Santos KF, Malaquias SG, Bachion MM, Silveira EA. Calf circumference: clinical validation for evaluation of muscle mass in the elderly. Rev Bras Enferm 2018; 71(2):322-328.

23. Gurney MJ, Jelliffe DB. Arm anthropometry in nutritional assessment: nomogram for rapid calculation of muscle circumference and cross-sectional muscle and fat areas. Am J Clin Nutr 1973; 26:912-915.

24. World Health Organization (WHO). Obesity: preventing and managing the global epidemic. Report of a WHO Consultation [Internet]. Geneva: WHO; 2000 [cited $2020 \mathrm{Fev} 23$ ]. Available from: https://www.who. int/nutrition/publications/obesity/WHO_TRS_894/ en/

25. Preto LSR, Gomes JRL, Novo AFMP, Mendes ER, Granero-Molina J. Efeitos de um Programa de Enfermagem de Reabilitação na Aptidão Funcional de Idosos Institucionalizados. Rev de Enfermagem Referência 2016; Série IV (8):55-63.

26. Placideli N, Castanheira ERL, Dias A, Silva PA, Carrapato JLF, Sanine PR, Machado DF, Medonça CS, Zarili TFT, Nunes LO, Monti JFC, Hartz ZMA, Nemes MIB.

27. Avaliação da atenção integral ao idoso em serviços de atenção primária. Rev Saude Publica 2020; 54(6):1-14.

28. Brasil. Ministério da Saúde (MS), Secretaria de Gestão Estratégica e Participativa, Departamento de Apoio à Gestão Participativa. Política Nacional de Saúde Integral da População Negra : uma política para o SUS. $2^{\mathrm{a}}$ ed. Brasília: MS; 2013. 
29. Brasil. Decreto ${ }^{\circ} 6.261$, de 20 de novembro de 2007. Dispõe sobre a gestão integrada para o desenvolvimento da Agenda Social Quilombola no âmbito do Programa Brasil Quilombola, e dá outras providências. Decreto $n^{\circ} 6.261 .2007,20$ nov.

30. Silva NDA, Menezes TN, Melo RLP, Pedraza DF. Força de preensão manual e flexibilidade e suas relações com variáveis antropométricas em idosos. Rev Assoc Med Bras 2013; 59(2):128-135.

31. Marques KM, Ferreira MPN, Freitas TI, Goulart RMM, Aquino RC, Previdelli ÁN. Evaluation of dynapenia in the elderly in São Caetano do Sul, São Paulo, Brazil. Fisioterapia em Movimento 2019; 32(E003218): $1-10$.

32. Lima TR, Silva DAS, Kovaleski DF, González-Chica DA. Associação da força muscular com fatores sociodemográficos e estilo de vida em adultos e idosos jovens no Sul do Brasil. Cien Saude Colet 2018; 23(11):3811-3820.

33. Ribeiro KG, Andrade LOM, Aguiar JB, Moreira AEMM, Frota AC. Education and health in a region under social vulnerability situation: Breakthroughs and challenges for public policies. Interface 2018; 22:1387-1398.

34. Ikegami ÉM, Souza LA, Tavares DMDS, Rodrigues LR. Capacidade funcional e desempenho físico de idosos comunitários: um estudo longitudinal. Cien Saude Colet 2020; 25(3):1083-1090.

35. Pavasini R, Guralnik J, Brown JC, di Bari M, Cesari M, Landi F, Vaes B, Legrand D, Verghese J, Wang C, Stenholm S, Ferrucci L, Lai JC, Bartes AA, Espaulella J, Ferrer M, Lim JY, Ensrud KE, Cawthon P, Turusheva A, Frolova E, Rolland Y, Lauwers V, Corsonello A, Kirk GD, Ferrari R, Volpato S, Campo G. Short Physical Performance Battery and all-cause mortality: Systematic review and meta-analysis. BMC Med 2016; 14(215):1-9.
36. Lenardt MH, Carneiro NHK, Betiolli SE, Binotto MA, Ribeiro DK de MN, Teixeira FFR. Factors associated with decreased hand grip strength in the elderly. Esc Anna Nery - Rev Enferm [periódico na Internet]. 2016 [acessado 2020 jan 12]; 20(4):e20160082 [cerca de 10p.]. Disponível em:https://doi.org/10.5935/14148145.20160082.

37. Garbaccio JL, Tonaco LAB, Estevão WG, Barcelos BJ. Envelhecimento e qualidade de vida de idosos residentes da zona rural. Rev Bras Enferm 2018;71(Supl. 2):776-784.

38. Silva N de A, Pedraza DF, Menezes TN de. Desempenho funcional e sua associação com variáveis antropométricas e de composição corporal em idosos. Cien Saude Colet 2015; 20(12):3723-3732.

39. Lenardt MH, Binotto MA, Carneiro NHK, Cechinel C, Betiolli SE, Lourenço TM. Handgrip strength and physical activity in frail elderly. Rev Esc de Enfermagem da USP 2016; 50(1):86-92.

40. Bezerra V, Andrade A, César C, Caiaffa W. Domínios de atividade física em comunidades quilombolas do sudoeste da Bahia , Brasil : estudo de base populacional. Cad Saude Publica 2015; 31(6):1213-1224.

Artigo apresentado em 20/11/2020

Aprovado em 23/05/2021

Versão final apresentada em 25/05/2021

Editores-chefes: Romeu Gomes, Antônio Augusto Moura da Silva 